\title{
Cost Sharing in Chains and Other Fixed Trees
}

\author{
by \\ Jens Leth Hougaard, \\ Mich Tvede \\ and \\ Lars Peter Østerdal
}

Discussion Papers on Business and Economics

No. $12 / 2013$

FURTHER INFORMATION

Department of Business and Economics

Faculty of Business and Social Sciences University of Southern Denmark Campusvej 55 DK-5230 Odense M Denmark

Tel.: +4565503271

Fax: +4565503237

E-mail: lho@sam.sdu.dk http://www.sdu.dk/ivoe 


\title{
Cost Sharing in Chains and Other Fixed Trees
}

\author{
Jens Leth Hougaard* Mich Tvede ${ }^{\dagger}$ \\ Lars Peter Østerdal ${ }^{\ddagger}$
}

September 2, 2013

\begin{abstract}
We consider a cost sharing problem among agents on a line. The problem is closely related to the classic airport game, but in our model agents are characterized by their location, rather than their needed runway length. We characterize a family of cost allocation rules in which agents pay a share of the incremental costs as well as any debt from upstream agents, with the Bird rule (where agents pay their full incremental cost) and the 'free rider' rule (where the terminal agent pays everything) as the two extreme cases. We also extend the analysis to cost sharing among agents located on a fixed tree structure.
\end{abstract}

Keywords: Airport game, Cost allocation, Axiomatic characterization, Bird Rule, Incremental cost sharing.

JEL: C71, D63.

*Corresponding author: Department of Food and Resource Economics, University of Copenhagen, Rolighedsvej 25, DK-1958 Frederiksberg C, Denmark, phone +45 353368 14, email: jlh@ifro.ku.dk

${ }^{\dagger}$ Newcastle University, UK, email: mich.tvede@ncl.ac.uk

${ }^{\ddagger}$ Department of Business and Economics, and COHERE, University of Southern Denmark, Denmark, email: lpro@sam.sdu.dk 


\section{Introduction}

The airport game is a classic cost sharing problem in the game theory literature (see, e.g., the surveys in [13] and [14]). The question posed is how the cost of a given runway is shared among a set of aircrafts which need different lengths of the runway in order to be served. Traditionally, it is modeled as a problem with a given set of agents (which we may think of as aircrafts) and each agent is characterized by a non-negative number interpreted as the needed length (cost) of the runway. A solution describes how the cost of a runway that satisfies the need of the agent with the highest demand (and therefore satisfies the need of all agents) is divided among the agents in any such situation.

While the airport model has proved useful for capturing many practical cases ([10]) and providing a case for applications of cooperative game theoretical solutions with especially nice properties ([8], [9], [3], [12]), the classical interpretation and its associated solutions is also somewhat narrow. For instance, when agents are sharing the costs of washing staircases, elevator installation, or scaffolding in apartment buildings, it is relevant to take into account the space utilized by the agents in the structure, and not only the maximal length needed.

In this paper we consider a problem of allocating the cost of a shared facility geometrically described as a line. Agents are characterized by their location on the line, rather than their needed length of the line as in the classical airport game, and their utility may depend on both the distance to the 'root' (point 0 on the line) as well as the size of the section of the line which they occupy.

In particular, we investigate a single-parameter family of allocation rules where agents' payments are based on shares of their incremental costs as well as any 'debt' from prior agents - to be dubbed the sequential $\lambda$-contribution rules. This family encompasses the so-called Bird rule (where each agent pays the cost of the adjacent edge in the direction of the root; see [2]) and the 'free rider' rule (where terminal agents share the total cost) as extremes.

We provide an axiomatic characterization of sequential $\lambda$-contribution rules and further single out the two extreme rules in the family: The Bird rule 
and the 'free rider' rule. The essential feature of the sequential $\lambda$-contribution rules is that agents' payments are independent of the presence of downstream agents both with respect to their costs and their number. This is in contrast to the sequential equal contributions rule, where agents' cost shares depend on the number of downstream agents.

We also extend our analysis to fixed tree structures and show how our axioms can be adapted to this more general setting by generalizing our characterization results.

\section{The chain model}

We study cost allocation in a variable population model. The universe of agents is identified with the set of natural numbers $\mathbb{N}$. Let $\mathcal{N}$ be the set of all finite non-empty subsets of $\mathbb{N}$.

A chain cost allocation problem is a pair $(N, C)$ where $N \in \mathcal{N}$ is a set of agents and $C=\left\{C_{i}\right\}_{i \in N} \in \mathbf{R}_{++}^{N}$ is a vector of agent characteristics such that $i>j \Rightarrow C_{i}>C_{j}$ for $i, j \in N$. A possible interpretation could be that $C_{i}$ is the location on a line of agent $i$; i.e., a distance from the reference point 0 (the root). Note that this interpretation differs from the usual airport model where $C_{i}$ is interpreted as the stand-alone cost of agent $i$; i.e., the cost of building a runway (of length $C_{i}$ ) for aircraft type $i$, see, e.g., [10]. Let $\mathcal{C}$ denote the set of such problems. With $N=\{1, \ldots, n\}$, some examples could be:

- The line is a time line. For instance, $\left[0, C_{n}\right]$ could be the total range of opening hours of a given airport facility (e.g., a gate), and $\left[C_{i-1}, C_{i}\right]$ could be the time slot assigned to airline $i$.

- The reference point 0 is a source of noise (or some other bad generating increasing disutility the closer the agent gets to 0$)$, and $\left[C_{i-1}, C_{i}\right]$ is the spacial location occupied by agent $i$.

In all the cases we consider, including those exemplified above, agents are associated with locations on a line and have to share the total cost of the situation. Assuming a one-to-one correspondence between distance and cost, 
the total cost $\max _{j \in N}\left\{C_{j}\right\}$ must be allocated among the agents involved taking into account that agents are concerned with the distance from 0 to their location $C_{i}$ as well as their increment $C_{i}-C_{i-1}$.

Generally, a cost allocation rule assigns a vector of cost shares $\phi(N, C) \in$ $\mathbb{R}^{N}$ to each problem $(N, C)$ in $\mathcal{C}$ satisfying budget balance, $\sum_{i \in N} \phi_{i}(N, C)=$ $\max _{j \in N}\left\{C_{j}\right\}$; boundedness, $0 \leq \phi_{i}(N, C) \leq C_{i}$ for all $i \in N$; and anonymity. 1

In the rest of the paper we assume (without loss of generality) that a game with $n$ players has the player set $N=\{1, \ldots, n\}$. Thus, the total cost $C_{n}$ can be decomposed into the sum of incremental costs as illustrated below: The incremental cost of the first agent is $C_{1}$, the incremental cost of the second agent is $C_{2}-C_{1}$, and so forth.

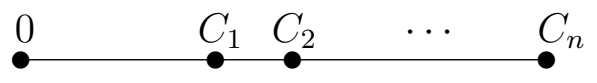

\section{Cost Allocation in Chains}

As mentioned above, it is inherent in the model that agents only care about the part of the chain between their location $C_{i}$ and the source 0 . Thus, a natural property to ask from the allocation rules is that the cost share of agent $i$ is independent of the exact location of any (downstream) agent $j$ with $C_{j}>C_{i}$. This 'independence' property is satisfied by many well-known rules; for instance, the rule where each agent pays for the increment that she is responsible for, i.e., where cost shares are given by

$$
x_{i}^{B}=C_{i}-C_{i-1},
$$

for all $i \in N$. The allocation defined by (1) is often named the Bird allocation, but it appears under many different names in the literature, e.g., the sequential full contributions rule ([14]).

\footnotetext{
${ }^{1}$ Formally, $\phi$ satisfies anonymity if, for any problem $(N, C) \in \mathcal{C}, g$ being a bijective function from $N$ to $N^{\prime}$ and $\left(N^{\prime}, C^{\prime}\right)$ being the game defined by $C_{g(i)}^{\prime}=C_{i}$ for all $i \in N$, we have $\phi_{g(i)}\left(N^{\prime}, C^{\prime}\right)=\phi_{i}(N, C)$.
} 
In this paper we will investigate a family of rules based on allocating incremental cost in the following way: The first agent pays a share $\lambda \in[0,1]$ of his incremental cost, the second agent pays a share $\lambda$ of his incremental cost as well as any remaining 'debt' from the first agent, etc., and the last agent, $n$, pays the residual. Hence, cost shares are determined recursively as $x_{1}^{\lambda}=\lambda C_{1}, x_{2}^{\lambda}=\lambda\left(C_{2}-C_{1}+(1-\lambda) C_{1}\right)=\lambda\left(C_{2}-x_{1}^{\lambda}\right)$ and so forth. That is,

$$
x_{i}^{\lambda}=\lambda\left(C_{i}-\sum_{j=1}^{i-1} x_{j}^{\lambda}\right),
$$

for all $i=1, \ldots, n-1$ and $x_{n}^{\lambda}=C_{n}-\sum_{j=1}^{n-1} x_{j}^{\lambda}$.

Note that we may rewrite (2) in the closed form expression

$$
x_{i}^{\lambda}=\lambda\left(C_{i}-C_{i-1}+(1-\lambda)\left(C_{i-1}-C_{i-2}\right)+\cdots+(1-\lambda)^{i-1} C_{1}\right),
$$

for $i=1, \ldots, n-1$ and

$$
x_{n}^{\lambda}=C_{n}-C_{n-1}+(1-\lambda)\left(C_{n-1}-C_{n-2}\right)+\cdots+(1-\lambda)^{i-1} C_{1} .
$$

Denote the corresponding family of allocation rules by $\phi^{\lambda}$. Clearly, $\phi^{1}$ is the Bird allocation rule (1). The opposite extreme, $\phi^{0}$, represents the case where the first $n-1$ agents free ride and the last agent pays the total cost.

A popular allocation rule in the literature (see, e.g., [7]) is the rule where all downstream agents share equally each incremental cost; i.e., cost shares are given by

$$
x_{i}^{S}=\sum_{k=1}^{i} \frac{C_{k}-C_{k-1}}{n+1-k} .
$$

This rule appears under many different names in the literature, e.g., the serial rule or the sequential equal contributions rule ([14]). This rule coincides with the Shapley value of a TU-game $(N, c)$ defined by $c(S)=\max _{i \in S}\left\{C_{i}\right\}$, for all $S \subseteq N$. Axiomatic characterizations can be found in [5], [11], [1], and [12].

Note that payments of the sequential equal contribution rule (3) depends on the number of downstream agents in contrast to payments of the sequential $\lambda$-contribution rules (2). This aspect will be further explored in the section below. 
Moreover, we observe that for $\lambda \neq 0$, the cost shares of the sequential $\lambda$ contribution rules (2) are not necessarily non-decreasing in the stand-alone cost (contrary to the cost shares of the sequential equal contribution rule (3)). In the traditional interpretation of the airport model this seems to be a quite undesirable feature of the $\lambda$-family. In other interpretations, however, incremental costs play a more prominent role, and hence an allocation reflecting solely stand-alone cost will not be reasonable.

Example 1: Consider three airline companies, A, B, and $\mathrm{C}$ sharing a gate which is open for 12 hours. Airline $\mathrm{A}$ is assigned time slot [0,5], $\mathrm{B}$ is assigned time slot $[5,7]$, and $\mathrm{C}$ is assigned time slot $[7,12]$. Customers of all airlines can use the gate facilities at any time during opening hours. Thus, an airline partially benefits from opening hours prior to its own time slot since its customers have access to the gate facilities. Now the airport has to allocate the total cost of having the gate open for 12 hours between the three airline companies. The figure below illustrates the situation.

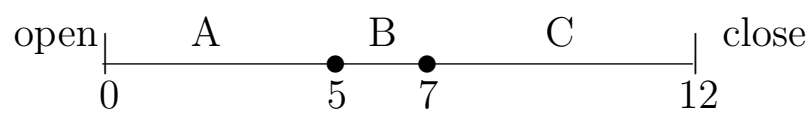

Assume a unit cost per hour of having the gate open such that the three airlines face the problem of sharing the total cost of 12 .

Using the Bird rule (1) we get $\left(x_{A}, x_{B}, x_{C}\right)=(5,2,5)$, whereas using the serial rule (3) we get $\left(x_{A}, x_{B}, x_{C}\right)=(5 / 3,8 / 3,23 / 3)$. Clearly, the Bird rule ignores the benefit from customers being able to use the gate facilities prior to their own departure. The serial rule, on the other hand, fails to reflect the length of the time slot used by each company.

Now the sequential $\lambda$-contribution rule (2) represents a kind of compromise with the Bird rule as one extreme case. Consider $\lambda=0.5$, which yields the cost shares $\left(x_{A}, x_{B}, x_{C}\right)=(2.5,2.25,7.25)$. Airline B now pays less than airline $\mathrm{A}$ but still more than its incremental cost. Note that airline $\mathrm{C}$ being the last and largest naturally pays most. 


\section{Axiomatics}

We now provide an axiomatic characterization of the family of allocation rules $\phi^{\lambda}$.

The last agent in the order plays a special role in the cost allocation problem because he determines the total cost of the group. For any other agent $i$, a natural requirement is that the cost share of $i$ shall only depend on the part of the problem that is determined by his own location as well as the location of the agents that are closer to the reference point 0 . Indeed, as there are no benefitting externalities for agent $i$ with respect to the number and locations of downstream agents, it seems reasonable to assume that the cost share of agent $i$ is independent of that. Formally,

Independence of Downstream Agents (IDA): For two cost allocation problems $(N, C)$ and $\left(N^{\prime}, C^{\prime}\right)$ with $\{1, \ldots, m+1\} \subset N \cap N^{\prime}$ and $C_{i}^{\prime}=C_{i}$ for all $i \leq m$

$$
\phi_{i}\left(N^{\prime}, C^{\prime}\right)=\phi_{i}(N, C)
$$

for all $i \leq m$.

In words, if two cost allocation problems coincide for a lower coalition of agents with no agent being the last agent, then these agents should pay the same. IDA is clearly satisfied by the sequential full contributions rule (as well as the rest of the $\lambda$-contribution rules, $\phi^{\lambda}$ ), but it is not satisfied by the sequential equal contributions rule because the cost shares depend on the total number of agents in the chain.

For the next axiom we need to define a reduced cost profile. Let $n \geq 2$ and consider the cost profile $C$, and let $\phi(N, C)$ be the associated cost allocation. Then excluding agent 1 results in a new cost profile $C_{-1}^{\phi}$ for the reduced problem where agent 1's cost share, $\phi_{1}(N, C)$, is subtracted from the cost of all the remaining agents; i.e.,

$$
C_{-1}^{\phi}=\left(C_{2}-\phi_{1}(N, C), \ldots, C_{n}-\phi_{1}(N, C)\right) .
$$

We now require that the allocation among agents $\{2, \ldots, n\}$ is unchanged by exclusion of agent 1. Formally, 
First-Agent Consistency (FAC): For all cost allocation problems $(N, C)$

$$
\phi_{i}\left(N \backslash\{1\}, C_{-1}^{\phi}\right)=\phi_{i}(N, C)
$$

for all $i \geq 2$.

This axiom is analyzed in [12]. They show that several rules satisfy FAC; for example both the sequential equal- and the sequential full contributions rule, and all rules satisfying FAC result in core allocations.

Finally, we consider the property of scale covariance stating that if costs are scaled by a factor $\alpha$, so is the solution.

Scale Covariance (SC): For all cost allocation problems $(N, C)$

$$
\phi(N, \alpha C)=\alpha \phi(N, C)
$$

for all $\alpha>0$.

Scale Covariance is a relatively mild condition, which is satisfied by most well known rules; e.g. both the sequential equal- and the sequential full contributions rule.

Theorem 1: A cost allocation rule $\phi$ satisfies IDA, FAC, and $S C$ if and only if $\phi(N, C)=\phi^{\lambda}(N, C)$.

Proof: It is easy to see that $\phi^{\lambda}$ satisfies IDA and SC. To see that $\phi^{\lambda}$ satisfies FAC, let $x_{i}=\phi_{i}^{\lambda}(N, C)$ and $\widetilde{x}_{i}=\phi_{i}^{\lambda}\left(N \backslash\{1\}, C_{-1}^{\phi}\right)$. Then $\widetilde{x}_{2}=\lambda\left(C_{2}-x_{1}\right)=$ $x_{2}$. For $3 \leq i<n$, we have $\widetilde{x}_{i}=\lambda\left(C_{i}-x_{1}-\sum_{j=2}^{i-1} \widetilde{x}_{j}\right)$. Thus, by induction $\widetilde{x}_{i}=x_{i}$ and $\widetilde{x}_{n}=C_{i}-x_{1}-\sum_{j=2}^{n-1} \widetilde{x}_{j}=x_{n}$. Thus, $\widetilde{x}_{i}=\phi_{i}^{\lambda}(N, C)$ for all $i \geq 2$.

Consider the reverse statement. First, let $N=\{1\}$ and $C=C_{1}$. By budget balance, $\phi_{1}(N, C)=C_{1}=\phi_{1}^{\lambda}(N, C)$. Next, add a second agent with $C_{2}>C_{1}$. Let $N^{\prime}=\{1,2\}$ and $C^{\prime}=\left(C_{1}, C_{2}\right)$. Then $\phi_{1}\left(N^{\prime}, C^{\prime}\right) \in$ $\left[0, C_{1}\right]$ so $\phi_{1}\left(N^{\prime}, C^{\prime}\right)=\lambda C_{1}=\phi_{1}^{\lambda}\left(N^{\prime}, C^{\prime}\right)$ for some $\lambda \in[0,1]$. By IDA, $\lambda$ is independent of $C_{2}$. Moreover, $\lambda$ is independent of $C_{1}$. To see this, suppose to the contrary that we have $C^{\prime}=\left(C_{1}, C_{2}\right)$ and $\tilde{C}=\left(\tilde{C}_{1}, \tilde{C}_{2}\right)$ with $C_{2}=\tilde{C}_{2}$ and $\phi_{1}\left(N^{\prime}, C^{\prime}\right)=\lambda C_{1}$ and $\phi_{1}\left(N^{\prime}, \tilde{C}\right)=\widetilde{\lambda} \widetilde{C}_{1}$ with $\lambda \neq \widetilde{\lambda}$. Then, by SC, 
$\phi_{1}\left(N^{\prime}, \frac{\widetilde{C}_{1}}{C_{1}} C\right)=\frac{\widetilde{C}_{1}}{C_{1}} \lambda C_{1}=\lambda \widetilde{C}_{1} \neq \widetilde{\lambda} \widetilde{C}_{1}$, contradicting that $\lambda$ is independent of $C_{2}$. Now by budget balance, $\phi_{2}\left(N^{\prime}, C^{\prime}\right)=C_{2}-\phi_{1}\left(N^{\prime}, C^{\prime}\right)=\phi_{2}^{\lambda}\left(N^{\prime}, C^{\prime}\right)$.

Next, suppose there is $\lambda$ such that $\phi=\phi^{\lambda}$ for all problems with up to $k$ agents, $k \geq 2$. Now, consider the problem $\left(N^{k}, C^{k}\right)$ with $N^{k}=\{1, \ldots, k\}$ and $C^{k}=\left\{C_{1}, \ldots, C_{k}\right\}$ and add an agent $k+1$ such that $C_{k+1}>C_{k}$. By IDA, $\phi_{i}\left(N^{k+1}, C^{k+1}\right)=\phi_{i}\left(N^{k}, C^{k}\right)=\phi_{i}^{\lambda}\left(N^{k}, C^{k}\right)$ for all $i \leq k-1$. By FAC, $\phi_{k}\left(N^{k+1}, C^{k+1}\right)=\phi_{k}\left(N^{k+1} \backslash\{1\}, C_{-1}^{k+1, \phi}\right)$ and thus by the induction hypothesis $\phi_{k}\left(N^{k+1}, C^{k+1}\right)=\phi_{k}^{\lambda}\left(N^{k+1}, C^{k+1}\right)$. By budget balance

$$
\phi_{k+1}\left(N^{k+1}, C^{k+1}\right)=C_{k+1}-\sum_{i=1}^{k} \phi_{i}^{\lambda}\left(N^{k+1}, C^{k+1}\right)=\phi_{k+1}^{\lambda}\left(N^{k+1}, C^{k+1}\right) .
$$

Q.E.D.

Remark 1: Independence of the axioms: 1 . The sequential equal contributions rule satisfies FAC and SC, but not IDA; $2 . \phi^{\lambda}$, defined as in (2) but where $\lambda=C_{1} /\left(1+C_{1}\right)$, satisfies IDA and FAC, but not SC; and 3 . the rule for which $\phi_{i}(N, C)=\lambda\left(C_{i}-C_{i-1}\right)$ for $i=1, \ldots, n-1$ (where $C_{0}=0$ and $0<\lambda<1)$ and $\phi_{n}(N, C)=C_{n}-\sum_{j=1}^{n-1} \phi_{j}(N, C)$ satisfies IDA and SC, but not FAC.

The sequential full contributions rule $\phi^{1}$ can be singled out by replacing FAC and SC with a property stating that cost shares should be increasing in incremental costs. Formally,

Incremental Cost Ranking (ICR): For every cost allocation problem $(N, C)$

$$
\phi_{i}(N, C) \geq \phi_{j}(N, C)
$$

for all $i$ and $j$ with $C_{i}-C_{i-1} \geq C_{j}-C_{j-1}$.

Theorem 2: A cost allocation rule $\phi$ satisfies IDA and ICR if and only if it is the sequential full contributions rule $\phi=\phi^{1}$.

Proof: It is easy to see that $\phi^{1}$ satisfies IDA and ICR.

Consider the reverse statement. First, let $N=\{1\}$ and $C=C_{1}$. By budget balance, $\phi_{1}(N, C)=C_{1}=\phi_{1}^{1}(N, C)$. Next, add a second agent with 
$C_{2}>C_{1}$. Let $N^{\prime}=\{1,2\}$ and $C^{\prime}=\left(C_{1}, C_{2}\right)$. Then $\phi_{1}\left(N^{\prime}, C^{\prime}\right) \in\left[0, C_{1}\right]$ so $\phi_{1}\left(N^{\prime}, C^{\prime}\right)=\lambda C_{1}=\phi_{1}^{\lambda}\left(N^{\prime}, C^{\prime}\right)$ for some $\lambda \in[0,1]$. By IDA, $\lambda$ is independent of $C_{2}$. Now, assume that $\lambda<1$. Then by budget balance we may choose $C_{2}$ such that ICR is violated (for example $C_{2}=2 C_{1}$ ). Thus, $\lambda=1$ as desired. By repeated use of IDA we are done.

Q.E.D.

The 'free-rider' rule $\phi^{0}$ can be singled out by considering the classical ranking axiom stating that cost shares should be ranked as the ordering of the agents stand-alone costs. Formally,

Ranking (R): For every cost allocation problem $(N, C)$

$$
C_{i}<C_{j} \Rightarrow \phi_{i}(N, C) \leq \phi_{j}(N, C)
$$

for all agents $i$ and $j$.

Theorem 3: A cost allocation rule $\phi$ satisfies IDA and $R$ if and only if $\phi=\phi^{0}$.

Proof: It easy to see that $\phi^{0}$ satisfies both IDA and R. Consider the converse claim. By contradiction, suppose that there exists a problem $(N, C)$ and an agent $i \in N, i \neq n$, such that $\phi_{i}(N, C)=\epsilon>0$.

Now consider a new problem $\left(N^{\prime}, C^{\prime}\right)$, where $N^{\prime}=\{1, \ldots, n+x\}$ for some arbitrarily chosen $x>\frac{C_{n}+1}{\epsilon}$ and $C_{j} \in\left(C_{n}, C_{n}+1\right]$ for each $j>n$. By IDA $\phi_{i}\left(N^{\prime}, C^{\prime}\right)=\phi_{i}(N, C)=\epsilon$, and hence by $\mathrm{R}$ we get that $\phi_{j}\left(N^{\prime}, C^{\prime}\right) \geq \epsilon$ for $j>n$. Thus, $\sum_{j=n+1}^{n+x} \phi_{j}\left(N^{\prime}, C^{\prime}\right) \geq x \epsilon>C_{n}+1$, violating budget balance. We conclude that $\phi_{i}(N, c)=0$ for $i=1, \ldots, n-1$ Q.E.D.

\section{$5 \quad$ Extension to Tree Models}

The family of allocation rules $\phi^{\lambda}$ has a natural extension to cost allocation among agents connected to a root by a fixed tree. A fixed tree is a graph with no cycles such that all agents are connected to the root via a unique path. 


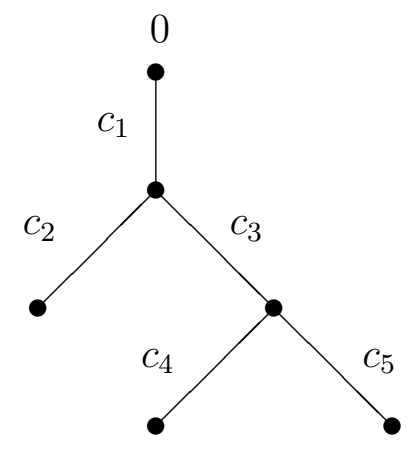

Imagine for example an airport terminal building with entrance at node 0 where different companies are responsible for different sections and have to share the total cost of maintenance, services or some other type of cost related to the flow of passengers through the facility.

Let $\left(N^{0}, E, c\right)$ be a fixed tree cost allocation problem, where $N^{0}=N \cup\{0\}$ is the set of agents including the root, $E$ is the set of edges in tree, and $c: E \rightarrow \mathbf{R}$ is an edge cost function. Given a tree, let $c_{i}$ denote the edge cost of agent $i$ (that is, the cost of the edge leaving the node $i$ on agent $i$ 's unique connected path to the root). The total cost of the tree is therefore $C=\sum_{i \in N} c_{i}$.

As before, a cost allocation rule assigns a vector of cost shares $\phi\left(N^{0}, E, c\right) \in$ $\mathbb{R}^{N}$ to each problem $\left(N^{0}, E, c\right)$ satisfying: budget balance, $\sum_{i \in N} \phi_{i}\left(N^{0}, E, c\right)=$ $C$; boundedness, $0 \leq \phi_{i}\left(N^{0}, E, c\right) \leq \sum_{i \in N_{i}} c_{i}$ for all $i \in N$, where $N_{i} \subseteq N$ is the set of nodes on agent $i$ 's unique connected path to the root (including node $i$ itself); and anonymity. ${ }^{2}$

Let $\delta(i)$ be the degree of node $i$ (i.e., the number of nodes directly connected to node $i$ in the tree). For $i \in N$, if $\delta(i)=1$, node $i$ is called a terminal node.

The family of allocation rules $\phi^{\lambda}$ defined in (2) easily extend to fixed trees: Agents that are not at terminal nodes pay a share $\lambda \in[0,1]$ of their edge $\operatorname{cost} c_{i}$ as well as an equal share (between successors of the node prior to i) of any remaining 'debt', and agents at terminal nodes pay their respective residuals. For an agent $i$, let $\{1, \ldots, i-1\}$ denote the nodes prior to node $i$

\footnotetext{
${ }^{2}$ Defined as the natural extension of the definition in footnote 1.
} 
on $i$ 's path to the root, ordered in terms of increasing distance to the root. Then we get

$$
x_{i}^{\lambda}=\lambda\left(c_{i}+\frac{(1-\lambda) c_{i-1}}{\delta(i-1)-1}+\cdots+\frac{(1-\lambda)^{i-1} c_{1}}{\prod_{z=1}^{i-1}(\delta(z)-1)}\right),
$$

for non-terminal agents. For terminal agents we get

$$
x_{i}^{\lambda}=c_{i}+\frac{(1-\lambda) c_{i-1}}{\delta(i-1)-1}+\cdots+\frac{(1-\lambda)^{i-1} c_{1}}{\prod_{z=1}^{i-1}(\delta(z)-1)} .
$$

Again, $\lambda=1$ gives us the sequential full contributions rule (the Bird allocation $x_{i}^{1}=c_{i}$ for all $i$ ), and $\lambda=0$ gives the rule where agents at terminal nodes pay all the relevant costs.

It is easy to extend the axioms of the chain model: In the context of trees it is natural to assume independence of sub-trees connected directly to the root (as there are no externalities between agents in different sub-trees). We dub such an axiom Tree Independence (TI).

An agent $j$ is a downstream agent for an agent $i$ if and only if $i$ is prior to $j$ on $j$ 's path to the root. For any non-terminal agent $i$, IDA now states that if two trees are similar except for the sub-tree of downstream agents of $i$, then $i$ should pay the same. We dub this new axiom Tree Independence of Downstream Agents (TIDA).

Next, consider extending First-Agent Consistency (FAC). In the context of trees, FAC only applies to trees where a single agent is connected directly to the root. Excluding this agent (agent 1) results in a new tree. For this tree the reduced edge cost profile is defined as follows: For an immediate successor of agent 1 the edge cost is changed to $c_{i}+\frac{c_{1}-\phi_{1}\left(N^{0}, E, c\right)}{\delta(1)-1}$, and for all other agents the edge cost is unchanged, $c_{i}$. Given this reduced edge cost profile we now require that cost shares of the other agents should remain unchanged by applying $\phi$ to the new tree. We dub this new axiom Tree First-Agent Consistency (TFAC).

The Scale Covariance axiom applies with the obvious changes.

We now state the following result.

Theorem 4: The result of Theorem 1 extends to the fixed tree model with the above restatements of the axioms. 
Proof: It is easy to see that $\phi^{\lambda}$ satisfies the axioms for any $\lambda$. We show the converse claim.

Define a $k$-node as the node for which the unique path to the root contains exactly $k$ edges. Consider an arbitrary tree and a 1-node $i$. If $i$ is a terminal node, then by TI and budget balance we have $\phi_{i}=c_{i}$. Otherwise, by TIDA and Theorem 1 there exists a $\lambda$ such that $\phi_{i}=\phi_{i}^{\lambda}$. Moreover, if there exists another non-terminal 1-node $j$, then by anonymity and TIDA $\phi_{j}=\phi_{j}^{\lambda}$ with the same $\lambda$.

By induction, assume that $\phi=\phi^{\lambda}$ for any tree with at most $n, k$-nodes, $k>1$. We wish to show that $\phi=\phi^{\lambda}$ for any tree with at most $n+1 k$-nodes, $k>1$. Consider now an arbitrary tree with $n k$-nodes and add to the tree an $n+1$ 'th $k$-node $j$. Without loss of generality we assume that $j$ is a terminal node. By TI we can disregard sub-trees connected to the root not containing $j$. By applying TFAC to the 1-node in the sub-tree containing $j$ 's path to the root we are back to a situation with at most $n k$-nodes, $k>1$ and we are done.

Q.E.D.

Remark 2: The two ways to single out the extreme rules $\phi^{1}$ and $\phi^{0}$, mentioned in Theorems 2 and 3, respectively, extend in a similar fashion to the fixed tree model.

Remark 3: As shown in [6], the cooperative game related to the fixed tree (where $c(S)$ is defined as the minimal cost needed to join all agents in coalition $S \subset N$ to the root via a connected subgraph of the fixed tree) is concave so the core is relatively large. Indeed, $\phi^{\lambda}$ results in core allocations for all $\lambda \in[0,1]$.

Example 2: Consider five airline companies A,B,C,D, and E located on a tree as shown below assuming that $c_{i}=1$ for each company $i$. 


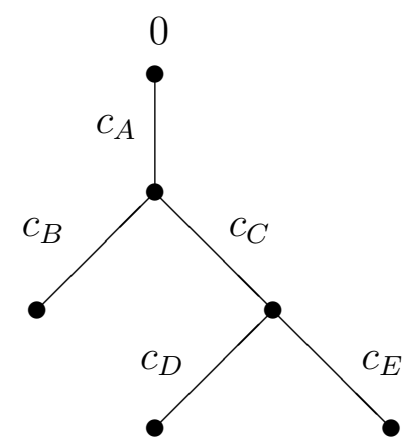

For $\lambda=0.5$ we get cost shares,

$\phi_{A}^{0.5}=0.5$

$\phi_{B}^{0.5}=1.25$

$\phi_{C}^{0.5}=0.625$

$\phi_{D}^{0.5}=\phi_{E}=1.3125$.

Thus, for $\lambda=0.5$ and identical edges cost, it is an advantage to be early in the tree as well as having many followers. As $\lambda \rightarrow 1$, the allocation approaches equal split. As $\lambda \rightarrow 0$, payments are pushed to companies B, D and E.

\section{Acknowledgements}

Financial support from the Danish Council for Strategic Research and The Danish Council for Independent Research | Social Sciences is gratefully acknowledged.

\section{References}

[1] Aadland, D. and V. Kolpin (1998), Shared irrigation costs: an empirical and axiomatic analysis, Mathematical Social Sciences, 35, 203-218.

[2] Bird, C.G. (1976), On cost allocation for a spanning tree: a game theoretic approach, Networks, 6, 335-350. 
[3] Branzei, R., E. Inarra, S. Tijs, and J.M. Zarzuelo (2006), A simple algorithm for the nucleolus of airport profit games, International Journal of Game Theory, 34, 259-272.

[4] Claus, A. and D.J. Kleitman (1973), Cost allocation for a spanning tree, Networks, 3, 289-304.

[5] Dubey, P. (1982), The Shapley value as aircraft landing fees - revisited, Management Science, 28, 869-874.

[6] Granot, D., M. Maschler, G. Owen and W.R. Zhu (1996), The kernel/nucleolus of a standard tree game, International Journal of Game Theory, 25, 219-244.

[7] Hougaard, J.L. (2009), An Introduction to Allocation Rules, Springer.

[8] Littlechild, S.C. (1974), A simple expression of the nucleolus in a special case, International Journal of Game Theory, 3, 21-29.

[9] Littlechild, S.C. and G. Owen (1973), A simple expression for the Shapley value in a special case, Management Science, 20, 370-372.

[10] Littlechild S.C. and G.F. Thompson (1977), Aircraft landing fees: a game theoretic approach, Bell Journal of Economics, 8, 186-204.

[11] Moulin, H. and S. Shenker (1992), Serial cost sharing, Econometrica, 60, 1009-1037.

[12] Potters, J. and P. Sudhölter (1999), Airport problems and consistent allocation rules, Mathematical Social Sciences, 38, 83-102.

[13] Sharkey, W.W. (1995), Network models in economics, in M.O. Ball et al., eds., Handbooks in OR and MS, Vol. 8, Elsevier.

[14] Thomson, W. (2007), Cost allocation and airport problems, Working paper, University of Rochester. 